\title{
Remoción de Semillas en Hábitats Transformados: Pogonomyrmex barbatus (Hymenoptera: Formicidae) y Cinco Especies de Cactáceas del Centro de México
}

by

Leticia Ríos-Casanova*, Héctor Godínez-Álvarez \& Gabriela Martínez Morales

\section{RESUMEN}

Una de las consecuencias de la realización de actividades humanas en las zonas semiáridas es la transformación del hábitat. En este trabajo se estudió el efecto de dicha transformación sobre la tasa de remoción de semillas, de cinco especies de catcáceas, por la hormiga Pogonomyrmex barbatus. El estudio se realizó en el Valle de Zapotitlán Salinas, una zona semiárida del centro de México. Se estudiaron tres sitios que han estado bajo diferentes actividades humanas: un campo de cultivo abandonado (CCA), una terraza con evidencia de actividades humanas actuales y una zona con pocas actividades dentro de un jardín botánico (JB). La hipótesis fue que el sitio que ha sido más transformado (CCA) tendría las tasas de remoción de semillas más bajas por presentar condiciones más adversas para la actividad de forrajeo de las hormigas. Los resultados mostraron que la transformación del hábitat afecta las tasas de remoción de las cinco especies de cactáceas estudiadas. Las tasas más bajas de remoción de semillas se encontraron en CCA y las más altas en TAH. Este resultado podría deberse a que TAH es un sitio muy heterogéneo que aún tiene algunas características que permiten una actividad de forrajeo alta por parte de las hormigas. También se encontró que la tasa de remoción de semillas varió ente las diferentes especies de cactáceas. Las semillas de Escontria chiotilla tuvieron la remoción más alta y las de Opuntia decumbens la más baja. Las diferencias en la remoción de semillas podrían estar asociadas con los cambios que las actividades humanas han ocasionado en la vegetación y en la superficie del suelo. Sin embargo la morfología externa de las semillas también debe ser considerada ya que en este estudio se encontró que las semillas más pequeñas fueron las más removidas.

UBIPRO, FES-Iztacala, Universidad Nacional Autónoma de México. Av. de los Barrios 1, Los Reyes Iztacala, Tlalnepantla 54090, Estado de México, México. leticiarc@campus.iztacala.unam.mx

* Autor para correspondencia 
Palabras clave: hormigas granívoras, Escontria chiotilla, México, Opuntia decumbens, Pachycereus hollianus, Stenocereus stellatus, Stenocereus pruinosus, tamaño de semillas, Valle de Tehuacán

\section{ABSTRACT}

One of the main consequences of human activities in semiarid zones is the transformation of habitats. In this work we studied the effect of this transformation on seed removal of five cacti species by the harvester ant Pogonomyrmex barbatus in the Tehuacán-Cuicatlán valley, a semiarid zone in central Mexico. Seed removal was quantified at three sites which have been under the effect of human activities: an abandoned crop field (CCA), a site with evidence of current human activities (TAH), and a site inside a botanic garden (JB). We hypothesized that sites which have been under intense human activities would have low rates of seed removal because they offer harsh conditions for harvester ants, reducing their foraging activity. Results showed that vegetation and surface soil characteristics of the sites studied are affecting the rates of seed removal of the five cacti species studied. The lowest seed removal rate was found at CCA, the most transformed site. In contrast with our hypothesis the highest seed removal was found at TAH, the site which represents the intermediate transformation condition, because this site still conserves some characteristics which permit intense foraging activity by harvester ants. We also found that the seed removal rate varied among the different cacti species studied. Seed of $E$. chiotilla had the highest removal rate, whereas $O$. decumbens had the lowest.

Differences in seed removal rate could be associated with the high heterogeneity found in sites with intermediate levels of transformation. Another factor that must be considered is the external morphology of seeds since smaller seeds presented highest removal rates.

Key words: cacti seeds, harvester ants, Escontria, México, Opuntia, Pachycereus, Stenocereus, seed size, Tehuacan valley

\section{INTRODUCCIÓN}

En las regiones áridas y semiáridas, la remoción de semillas por hormigas puede afectar la diversidad de la comunidad de plantas, por lo que esta interacción es importante para la estructura y funcionamiento de estos ecosistemas 
(Brown et al. 1986, Whitford 2002). Las hormigas consumen las semillas de una amplia variedad de especies, llegando a remover hasta $14 \mathrm{~g}$ de semillas en $12 \mathrm{~h}$ (Whitford 2002). Sin embargo, la remoción puede variar dependiendo de las características de los sitios en donde ésta ocurre (Whitford 2002). En este sentido, fenómenos como la fragmentación, la transformación del hábitat y el disturbio pueden estar afectando dicha interacción (Sassi et al. 1996; Kerley \& Whitford 2000; Nicolai et al. 2007).

La importancia de la remoción de semillas por hormigas ha sido relativamente bien documentada en desiertos extra-tropicales de Argentina, Australia, Chile, Estados Unidos, Israel y Sudáfrica (Marone et al. 2000; Pirk \& López de Casenave 2011). Por el contrario, la información sobre la importancia relativa de esta interacción en desiertos tropicales es prácticamente inexistente (Ibáñez \& Soriano 2004). Los estudios previamente realizados en las zonas semiáridas del centro de México (Valle de Tehuacán) (Guzmán 2004; Ríos-Casanova et al. 2004; Ríos-Casanova 2005; Ríos-Casanova et al. 2006) sugieren que las hormigas podrían tener un papel importante en la remoción de semillas en la región. Sin embargo, hasta el momento no existen estudios sobre esta interacción ni sobre la respuesta de las hormigas a la transformación del hábitat y sus consecuencias sobre la remoción de semillas.

La transformación del hábitat en las zonas semiáridas del centro de México ocurre principalmente debido a las actividades humanas que se realizan en estos lugares como la agricultura, la ganadería, la extracción de leña y la construcción de salinas. Dichas actividades han originado una disminución en la estructura de la vegetación, y un aumento de las áreas de suelo desnudo, por lo que los sitios más transformados presentan fuertes evidencias de erosión (López-Galindo et al. 2003; Muñoz-Iniestra 2008, Gaytán 2011).

Debido a estos cambios, es posible encontrar sitios con una vegetación muy poco estructurada que reciben una alta insolación y tienen una baja disponibilidad de semillas, en los que se esperaría registrar tasas muy bajas de remoción de semillas. Por el contrario, existen sitios con vegetación más estructurada y que por lo tanto se encuentran menos expuestos a la radiación solar y que ofrecen una mayor disponibilidad de recursos, en los cuales se esperaría que las tasas de remoción de semillas fueran más altas.

El presente trabajo se llevó a cabo en una porción del Valle de TehuacánCuicatlán, una zona semidesértica del centro de México en la que se ha 
reportado a la hormiga Pogonoyrmex barbatus como uno de los elementos granívoros más importantes (Ríos-Casanova et al.2004, Ríos-Casanova 2005; Ríos-Casanova et al. 2006; Guzmán-Mendoza et al. 2010).

Se analizó la remoción de semillas de cactáceas que son las plantas dominantes en el sitio de estudio, el cual es considerado un centro de diversidad de esta familia de plantas muchas de las cuáles son endémicas (Godínez-Alvarez \& Valiente-Banuet 1998). La remoción de las semillas de estas plantas se evaluó en tres sitios que tienen distintas características fisonómicas de la vegetación y del suelo, principalmente como producto de la realización de actividades humanas.

Se esperaba que las tasas de remoción de semillas por la hormiga $P$. barbatus fueran bajas en los sitios que como consecuencia de la realización intensa de actividades humanas tienen vegetación poco estructurada, mientras que los sitios que han recibido menos impacto por las actividades humanas y que tienen una vegetación más estructurada deberían tener tasas de remoción de semillas mayores debido a la mayor disponibilidad de recursos y a que las condiciones ambientales permiten a las hormigas periodos de actividad más amplios.

\section{MATERIALES Y MÉTODOS}

\section{Sitios de estudio}

El estudio se realizó en Zapotitlán de las Salinas, Puebla $\left(17^{\circ} 48^{\prime}-18^{\circ} 58^{\prime} \mathrm{N}\right.$, $\left.97^{\circ} 03^{\prime}-97^{\circ} 43^{\prime} \mathrm{W}\right)$, que se ubica dentro de la Reserva de la Biosfera del Valle de Tehuacán-Cuicatlán, el cual abarca la porción noreste del estado de Oaxaca y el sureste del estado de Puebla en el centro de México. El clima es seco con una estación de lluvias que ocurre entre mayo y septiembre. La precipitación media anual es de $412.4 \mathrm{~mm}$ y la temperatura media anual varía entre 17.6 y $23.7^{\circ} \mathrm{C}$ (Valiente 1991 ).

Se sabe que en este sitio existen aproximádamente 290 especies de angiospermas de las cuales 81 son cactáceas (Dávila et al. 1993). Los principales tipos de vegetación son el "matorral espinoso" con Mimosa luisana (Mimosaceae) y Prosopis laevigata (Mimosaceae) y las "tetecheras" dominadas por el cactus columnar Neobuxbaumia tetetzo (Cactaceae) (Osorio et al. 1996).

Para probar la hipótesis se realizaron experimentos de remoción de semillas por $P$. barbatus en tres sitios en los que los efectos de las actividades humanas son 
notables: 1) campo de cultivo abandonado (CCA), 2) jardín botánico (JB) y 3) terraza con evidencias de la práctica de varias actividades humanas (TAH). El CCA se caracteriza por ser un área con varios campos de cultivo que no han sido labrados en los últimos cinco años (Fig. 1a). No hay presencia de costras biológicas en la superficie del suelo y, alrededor de lo que fueron los campos, crecen algunos árboles como Pirul (Schinus mole) y Mezquite (Prosopis laevigata), algunas cactáceas del género Opuntia y columnares como Pachycereus hollianus, además de diferentes herbáceas anuales como Viguiera dentata, Ipomea violacea y algunas gramíneas (Oliveros 2000). El JB es un sitio que pertenece al jardín botánico "Helia Bravo Hollis". Predomina el cactus columnar Neobuxbaumia tetetzo y crecen una gran variedad de plantas asociadas a esta tetechera como la cactácea $P$. hollianus, y las leguminosas Mimosa luisana y Parkinsonia praecox (Osorio et al. 1996). La actividad humana en este sitio es principalmente el ecoturismo (Fig. 1b). La TAH presenta zonas con vegetación abundante y algunas áreas con escasa o ninguna cobertura vegetal, también se encuentran zonas cubiertas con costra biológica. Presenta especies como $P$. laevigata, Pachycereus hollianus, Opuntia decumbens y $O$ pilifera. Es evidente la presencia de actividades humanas como la extracción de leña y el cultivo de pitahaya (Hylocereus undatus) (Oliveros 2000; Fig. 1c).

En el cuadro 1 se presentan las características de cada uno de los sitios en términos de algunos indicadores ecológicos como porcentaje de cobertura vegetal, porcentaje de claros en la superficie del suelo, porcentaje de pedregosidad, etcétera. Los datos para CCA y TAH se obtuvieron de Gaytán (2011) mientras que los datos para JB se obtuvieron siguiendo la metodología de Herrick et al. (2005).
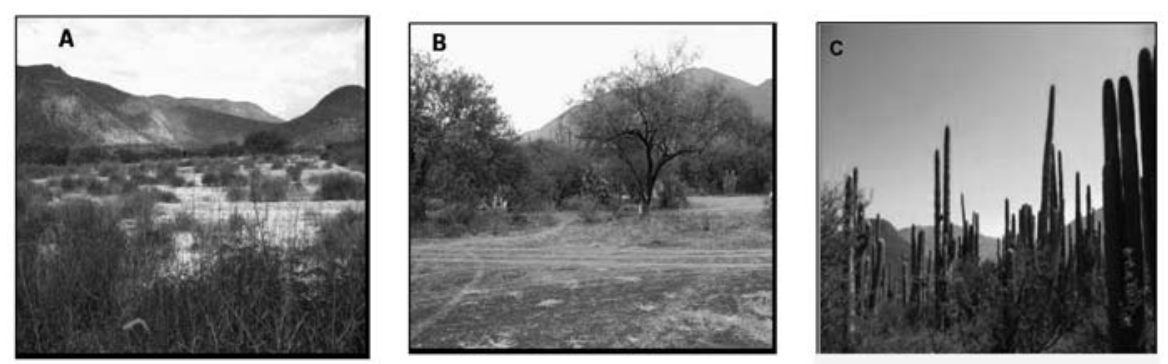

Figura 1. Sitios de estudio. A) Campo de cultivo abandonado, B) Terraza con actividades humanas, C) Jardín botánico. 
Cuadro 1. Características de los sitios estudiados. $\mathrm{CCA}=$ campo de cultivo abandonado, $\mathrm{TAH}=$ terraza con actividades humanas, JB = Jardín Botánico. Promedios \pm un error estándar.

\begin{tabular}{llll}
\hline \hline & CCA & TAH & JB \\
\hline Cobertura vegetal (\%) & $21.6 \pm 5.8$ & $39 \pm 13.4$ & $63.33 \pm 5.81$ \\
Suelo desnudo (\%) & $72.5 \pm 6.7$ & $13.33 \pm 8.0$ & $6.66 \pm 2.40$ \\
Rocas en superficie del suelo (\%) & $1.66 \pm 1.66$ & $0 \pm 0$ & $44.66 \pm 2.66$ \\
Hojarasca (\%) & $4 \pm 2.5$ & $36.6 \pm 3.6$ & $10 \pm 4.16$ \\
Obstrucción vertical por la vegetación (\%) & $20 \pm 6.5$ & $33 \pm 4.6$ & $57.88 \pm 6.83$ \\
Estabilidad del suelo & $3.2 \pm 0.24$ & $5.8 \pm 0.18$ & $4.83 \pm 0.55$ \\
Espacios en el dosel > 10 m (\%) & $23 \pm 13.9$ & $42.6 \pm 6.0$ & $28.58 \pm 5.6$ \\
Espacios en la superficie del suelo $>10 \mathrm{~m}(\%)$ & $59.3 \pm 8.7$ & $62.65 \pm 11.5$ & $40.9 \pm 5.9$ \\
\hline \hline
\end{tabular}

\section{Semillas}

Las semillas que se emplearon pertenecen a cinco especies de cactáceas que tienen una amplia distribución en el área de estudio además de ser dominantes: Pachycereus hollianus, Stenocereus pruinosus, S. stellatus, Escontria chiotilla y Opuntia decumbens. Estas especies se escogieron debido a que fructificaron durante el año de estudio (2009) y por lo tanto representaron un gran número de semillas disponibles para las hormigas. Todas estas cactáceas son endémicas de México, excepto $S$. pruinosus que también se encuentra en Centroamérica. La mayoría son cactáceas columnares a excepción de $O$. decumbens que es un arbusto cuyos cladodios crecen de forma rastrera.

Las semillas de las cinco especies de cactáceas se obtuvieron de al menos 25 frutos maduros elegidos al azar en diferentes sitios del Valle de Zapotitlán. Posteriormente se extrajo la pulpa la cual se lavó con agua corriente con ayuda de una malla que permitió el paso de la pulpa pero no de las semillas Una vez lavadas, se colocaron sobre papel absorbente a temperatura ambiente, y ya secas se guardaron en bolsas de papel para ser utilizadas en los experimentos de remoción.

\section{Experimentos de remoción}

Para cuantificar la remoción de semillas, en cada uno de los tres sitios se localizaron 5 nidos de la hormiga Pogonomyrmex barbatus. Alrededor de cada nido se colocaron cuatro cajas de Petri a $3 \mathrm{~m}$ de la entrada del nido y separadas $90^{\circ}$ una de la otra. En la superficie de dichas cajas se pegó un cuadro de papel lija en cuyo centro se dibujo un cuadro de $1 \mathrm{~cm}^{2}$, el cual sirvió para determinar el número de semillas removidas por las hormigas (Kaspari 1996). 
Dentro de cada cuadro se colocó una capa de semillas de una de las especies de cactáceas y se revisaron las cajas cada media hora para contar el número de semillas removidas y remplazar con más semillas hasta llenar el cuadro de $1 \mathrm{~cm}^{2}$ (Fig. 2).

Cuadro 2. Tamaños y características principales delas 5 especies de cactáceas utilizadas en los experimentos de remoción por la hormiga granivora $P$. barbatus.

\begin{tabular}{lllllll}
\hline \hline Especies & Largo & $\begin{array}{l}\text { Ancho } \\
\text { mayor }\end{array}$ & $\begin{array}{l}\text { Ancho } \\
\text { menor }\end{array}$ & Grosor & $\begin{array}{l}\text { Largo } \\
\text { del hilio }\end{array}$ & Color \\
\hline E. chiotilla & $1.53 \pm 0.02$ & $1.15 \pm 0.02$ & $0.88 \pm 0.02$ & $0.83 \pm 0.06$ & $0.62 \pm 0.02$ & Marrón brillante \\
P.hollianus & $2.66 \pm 0.04$ & $2.13 \pm 0.03$ & $1.82 \pm 0.04$ & $1.51 \pm 0.02$ & $1.54 \pm 0.03$ & Café obscuro \\
S.pruinosus & $2.28 \pm 0.07$ & $1.77 \pm 0.03$ & $1.44 \pm 0.02$ & $1.14 \pm 0.02$ & $0.89 \pm 0.02$ & Marrón \\
S. stellatus & $1.92 \pm 0.03$ & $1.41 \pm 0.03$ & $1.2 \pm 0.02$ & $0.93 \pm 0.02$ & $0.66 \pm 0.02$ & Marrón mate \\
O. decumbens & $3.1 \pm 0.03$ & $2.75 \pm 0.03$ & $1.89 \pm 0.06$ & $1.82 \pm 0.04$ & $0.38 \pm 0.02$ & Blanco opaco \\
\hline \hline
\end{tabular}

\section{CAJA DE PETRI CON $1 \mathrm{~cm}^{2}$ DE SEMILLAS}

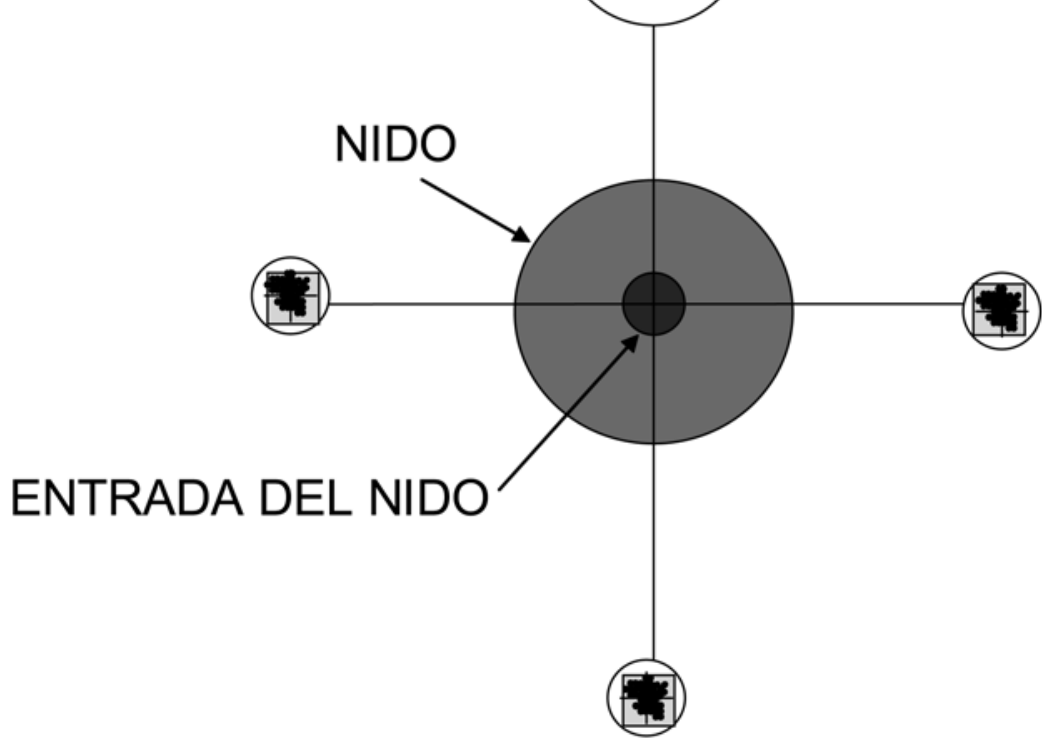

Figura 2. Disposición de las semillas ofrecidas alrededor de la entrada del nido de P. barbatus. 
Debido a las dificultades que presenta el conteo de semillas pequeñas en el campo, en cada revisión solamente se anotaba si 1/4, 1/2,3/4, o todas las semillas del cuadro de $1 \mathrm{~cm}^{2}$ habían sido removidas. Para conocer el número de semillas de cada especie de cactácea que fueron removidas, en el laboratorio se rellenó el cuadro de $1 \mathrm{~cm}^{2}$ con las semillas de cada una de las especies ofrecidas a las hormigas para contar el número de semillas que caben en el cuadro. Esta medición se hizo 20 veces para cada especie de semilla y se calculó un promedio. El número de semillas removidas de cada especie de cactácea se calculó multiplicando la fracción de semillas removidas en el campo por el número promedio de semillas que caben en el cuadro de $1 \mathrm{~cm}^{2}$.

Los experimentos de remoción se realizaron entre las 10 y las 15 horas (horario de verano) por dos días, haciendo un total de 10 horas de observación para cada especie. La remoción se midió como el número de semillas totales removidas por las hormigas, entre el número de horas de observación por especie (número de semillas removidas/hora).

\section{Relación tamaño-tasa de remoción.}

Para explorar si algunas de las características morfométricas de las semillas están relacionadas con las tasas de remoción encontradas, se midieron el largo, ancho mayor, ancho menor y grosor de las semillas, así como el ancho del hilio de 20 semillas de cada especie de cactácea y estas medidas se correlacionaron con la tasa de remoción de cada especie. Para este caso se calculó una tasa de remoción general, es decir, sin tomar en cuenta el sitio, ya que las semillas provienen de sitios con diferentes características y no fue posible diferenciar su origen. Los tamaños de las semillas se obtuvieron utilizando el programa Motic Mc Images plus 2.0 ML., el cuál permite realizar mediciones digitales de objetos observados en el microscopio estereoscópico.

\section{Análisis estadístico}

Las tasas de remoción para cada especie de semillas fueron comparadas entre sitios utilizando una prueba de Kruskal Wallis siendo la tasa de remoción la variable de respuesta y los sitios la fuente de variación. Las medias se compararon entre si utilizando la prueba de $q$ (Zar 1996). Estos análisis estadísticos se realizaron utilizando el programa SPSS 9.0 para Windows.

Para conocer la relación entre las medidas de las semillas y su tasa de remoción se hicieron correlaciones lineales para cada parámetro utilizando el programa JMP 3.1.2 (SAS Institute). 


\section{RESULTADOS}

Los resultados mostraron que las características fisonómicas de los sitios debidas principalmente al efecto de las actividades humanas, afectan la tasa de remoción de las semillas por la hormiga $P$. barbatus. La menor y mayor tasas de remoción se registraron en CCA y TAH, respectivamente. La tasa de remoción en JB tendió a presentar valores intermedios (Fig. 3).

La tasa de remoción también varió dependiendo de la especie de cactácea (Fig. 3). La remoción de las semillas de $S$. pruinosus y E.chiotilla fue significativamente diferente entre sitios $\left(C h i^{2}=7.02, P=0.03\right.$ para ambas especies) siendo mayor en TAH aunque no difirió de la tasa encontrada para JB, mientras que en CCA no se registró remoción. Este patrón fue similar para las especies $S$. stellatus y $O$. decumbens, aunque no se encontraron diferencias significativas entre los sitios $\left(C h i^{2}=3.37, P=0.18 ; C h i^{2}=4.61, P=0.09\right.$ respectivamente). Las semillas de $P$. hollianus fueron removidas en los tres sitios, sin embargo no se encontró efecto significativo del sitio $\left(\mathrm{Chi}^{2}=0.43\right.$, $P=0.8$ )

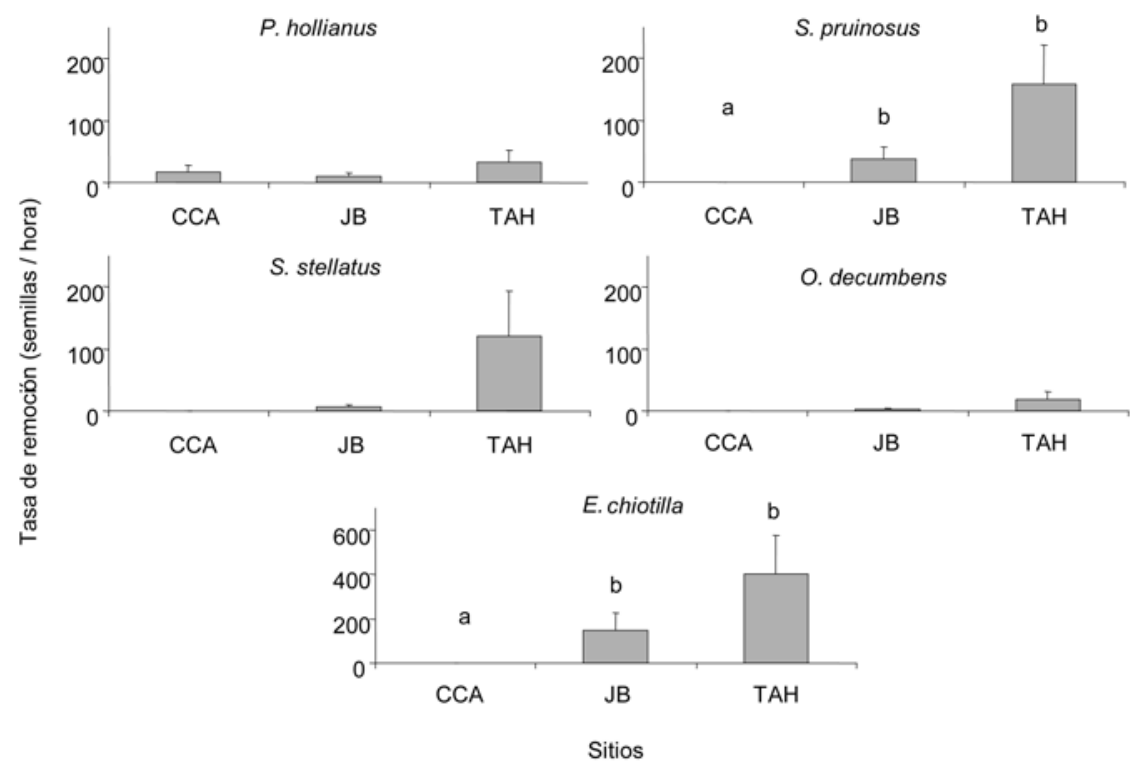

Fig. 3. Tasa de remoción de semillas por $P$. barbatus para cinco especies de cactáceas en tres sitios (CCA $=$ Campo de cultivo abandonado, JB $=$ Jardín botánico, $\mathrm{TAH}=$ Terraza con actividades humanas) de Zapotitlán de las Salinas, Puebla. Medias marcadas con letras diferentes son estadísticamente diferentes (prueba de $q, P<0.05$ ). 
Únicamente la longitud del ancho menor de las semillas se correlacionó con su tasa de remoción $\left(R^{2}=0.75, F=9.23, P=0.05\right.$; Fig. 4). Para las otras medidas de la semilla las correlaciones no fueron significativas (largo: $R^{2}=$ $0.7, F=7.0, P=0.07$; ancho mayor: $R^{2}=0.61, F=4.6, P=0.12$; grosor; $R^{2}=0.56, F=3.8, P=0.15$; largo del hilio: $R^{2}=0.04, F=0.15, P=0.73$; $P=0.85)$.

\section{DISCUSIÓN}

Los resultados obtenidos indican que la remoción de semillas llevada a cabo por la hormiga Pogonomyrmex barbatus puede estar afectada por las transformaciones que han ocurrido en su hábitat como resultado de factores biológicos y de las actividades humanas que se llevan a cabo en la zona de estudio.

Las características propias de cada sitio, medidas por medio de indicadores, mostraron que los tres sitios se han transformado de manera diferente. En el sitio CCA se registró la menor cobertura vegetal, menor porcentaje de obstrucción visual ocasionada por la vegetación, menor estabilidad del suelo y mayor porcentaje de espacios con suelo desnudo. Todas estas características indican que este es el sitio con mayor grado de transformación, lo que puede ser resultado de la intensa actividad agrícola realizada en el pasado.

De acuerdo con la hipótesis planteada, en este sitio se registraron las

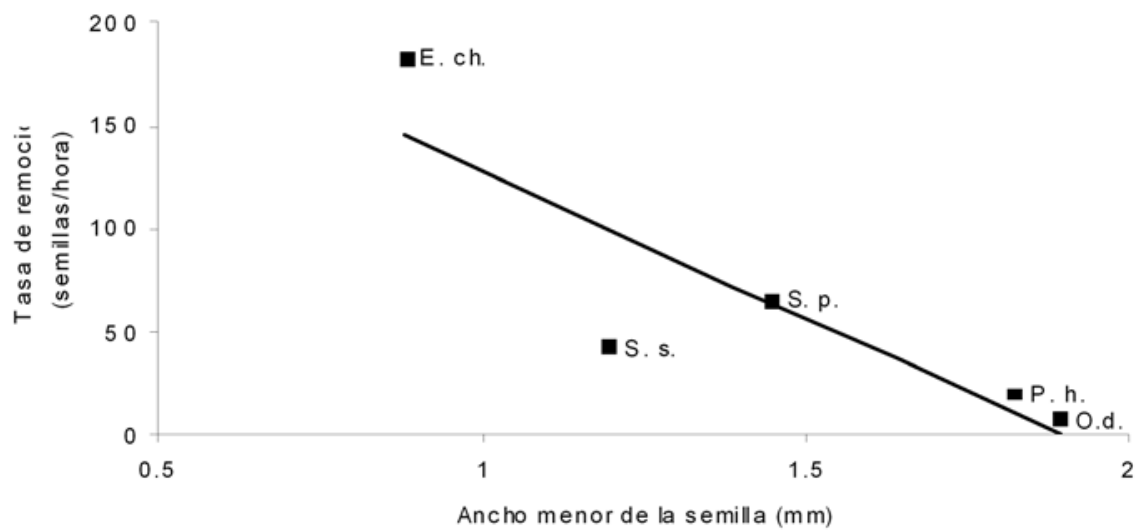

Figura 4. Regresión lineal entre el tamaño del ancho menor de las semillas y su tasa de remoción por la hormiga $P$. barbatus en Zapotilán de las Salinas, Puebla. Ecuación de la recta: $y=-144.01 x$ +271.7 . 
menores tasas de remoción de semillas para todas las especies de cactáceas estudiadas. Únicamente se observó la remoción de semillas de $S$. stellatus y $P$. hollianus y sus tasas de remoción fueron tan bajas que no sobrepasaron las 0.3 y 17 semillas por hora respectivamente.

Los sitios con poca cobertura vegetal como el CCA, se encuentran expuestos a la radiación directa del sol por lo que son sitios en los que las hormigas están expuestas a las altas temperaturas y a la desecación (López et al. 1992). Se ha demostrado que la temperatura es uno de los factores más importantes en la regulación de las actividades de las hormigas (Whitford \& Ettershank 1975; MacKay \& Mackay,1989; Hölldobler \& Wilson 1990). Particularmente para las hormigas del género Pogonomyrmex se ha documentado que tienen su mayor actividad entre los 20 y $45^{\circ} \mathrm{C}$ y no realizan ninguna actividad por arriba de los $60^{\circ} \mathrm{C}$ (MacKay y MacKay 1989; Hölldobler \& Wilson 1990; Ríos-Casanova 2005). Sitios como el CCA pueden alcanzar temperaturas por arriba de los $50^{\circ} \mathrm{C}$ en la superficie del suelo (observación personal) por lo que representa un lugar poco propicio para la búsqueda y remoción de las semillas ofrecidas durante los experimentos ya que predominan los sitios desprovistos de vegetación. Por la misma razón, estas hormigas son vulnerables al ataque de depredadores. Uno de los principales depredadores de P. barbatus son las lagartijas del género Sceloporus. En el sitio CCA, se observaron lagartijas de este género y se ha documentado que una gran proporción de la dieta de estas lagartijas en el Valle de Zapotitlán son las hormigas P. barbatus (SerranoCardozo et al. 2008).

A pesar de lo anterior, en este sitio se observó forrajear a las hormigas en sitios cubiertos por los arbustos anuales que se encuentran alrededor de los campos de cultivo, entre ellas algunos pastos y asteráceas cuyas semillas eran ocasionalmente removidas por las hormigas.

El sitio JB fue considerado como el menos deteriorado debido a que presentó mayor cobertura de la vegetación y por lo tanto mayor obstrucción visual provocada por la misma. Igualmente, presentó pocos espacios en el dosel y el menor porcentaje de suelo desnudo; además este sitio presentó poca evidencia de la práctica de actividades humanas a excepción del ecoturismo. Los resultados obtenidos difieren de la hipótesis planteada, ya que en este sitio se esperaba encontrar las mayores tasas de remoción. Sin embargo, los valores de remoción encontrados, aunque fueron mayores que en el CCA, 
no fueron los más altos. En este sitio hubo remoción de semillas de todas las especies siendo $E$. chiotilla la especie con la mayor tasa de remoción (149 semillas por hora).

Se esperaba que en JB ocurriera la mayor remoción ya que por sus características, tiene el mayor porcentaje de sitios protegidos de la incidencia solar directa, lo que permitiría a las hormigas forrajear durante rangos mayores de tiempo provocando tasas de remoción altas (Hensen 2002). Sin embargo las tasas de remoción encontradas podrían deberse a que las hormigas forrajearon otras semillas que estaban disponibles en ese momento como por ejemplo semillas de gramíneas. También se ha sugerido que la dirección en la que las hormigas forrajean está orientada por marcadores químicos que las hormigas exploradoras de la colonia dejan en la superficie del suelo al detectar un recurso (Gordon 2002). El sitio JB fue el sitio con el mayor porcentaje de rocas en la superficie, lo cual podría haber dificultado la búsqueda y el transporte de las semillas de las cactáceas ofrecidas, así como la comunicación química entre forrajeras.

Un estudio previo realizado en el JB señaló que en este sitio se observó la presencia de depredadores arácnidos cerca de los nidos, sugiriendo que habría una fuerte presión por depredación sobre las hormigas P. barbatus (Guzmán 2004). Lo anterior podría estar disminuyendo los periodos de actividad de las hormigas en este sitio y por lo tanto sus tasas de remoción de semillas.

Elsitiodenominado terraza con actividades humanas(TAH), fuellamadoasí por presentar evidencias de que ahí se realizan actividades como extracción de leña y cultivo de la cactácea Hylocereus undatus, entre otras. La transformación de este sitio debida a la realización de dichas actividades le ha conferido una alta heterogeneidad, ya que existen zonas con mucha insolación, pero también zonas sombreados por arbustos y árboles, zonas cubiertos de costra biológica y zonas con suelo desnudo.

Aquí encontramos la mayor tasa de remoción para todas las especies de cactáceas estudiadas, desde 32 semillas por hora para $O$. decumbens, hasta 400 semillas por hora para E. chiotilla.. Este patrón podría deberse a que es un sitio muy heterogéneo en el que hay sitios sombreados con temperaturas que pueden ser toleradas por las hormigas, sitios con diferentes texturas en el suelo, lo cual puede reducir las temperaturas del mismo, y una gran posibilidad de encontrar semillas de diferentes recursos en diferentes momentos. 
Igualmente, la alta heterogeneidad podría estar proveyendo a las forrajeras de sitios en los que pueden escapar de la depredación.

Se ha sugerido que la granivoría es resultado de cambios en la estructura y composición de los ensamblajes tanto de plantas como de la comunidad de granívoros en general y que los factores que ocasionan disturbio son una fuente de heterogeneidad lo cual repercute en las tasas de remoción llevadas a cabo por los granívoros (Wilby \& Shachak 2000; Sassi et al. 2006; Lomov et al. 2009).

En este sitio(TAH) la semilla más removida fue E. chiotilla yaque se encontró que $P$. barbatus removió cerca de 400 semillas por hora. En otros estudios se ha encontrado que las hormigas del género Pogonomyrmex pueden remover hasta 30 semillas en un día (Mull \& MacMahon 1997), por lo que las tasas encontradas en este estudio indican una remoción sumamente elevada.

En lo que respecta a las características de las semillas y sus tasas de remoción los resultados indicaron que las semillas más pequeñas tienen las tasas de remoción más altas. Entre las características de las semillas sobre las cuales se ha sugerido que opera la selectividad de las hormigas se encuentran el tamaño, la morfología, el contenido de agua y la composición nutricional, así como las habilidades de las hormigas para reconocer el alimento (Ibáñez \& Soriano 2004).

En este estudio solo se consideraron aspectos relacionados con el tamaño de las semillas y se encontró que las semillas menos removidas fueron las de O. decumbens. Estas semillas, además de ser las más grandes no presentan ornamentaciones evidentes en la testa, lo que podría estar dificultando su manipulación y traslado por parte de $P$. barbatus. Se ha documentado que el transporte de semillas con superficies lisas es más difícil (Pirk, 2002). También se ha documentado que las obreras de $P$. barbatus prefieren las semillas con prolongaciones y ornamentaciones que facilitan su manejo (Pulliam \& Brand 1975). Las semillas de E. chiotilla fueron las semillas más removidas en este estudio y, aunque no tienen prolongaciones, tienen una constricción que corresponde al ancho menor, que podría estar facilitando el manejo por parte de las hormigas. Además estas semillas son las más pequeñas, lo que puede facilitar su transporte. Las semillas de las otras especies, P. hollianus, $S$. stellatus y $S$. pruinosus, son semillas más grandes que, aunque no presen- 
tan prolongaciones, si fueron removidas por $P$. barbatus aunque con menor frecuencia que las de E. chiotilla.

Otro factor que podría estar afectando directamente la preferencia de las semillas por las hormigas es su contenido nutricional (Kelrick et al. 1986) y la presencia de compuestos tóxicos (Carroll \& Janzen 1973). Aunque en este estudio no se midió ninguna variable relacionada con la química de las semillas, datos obtenidos en la literatura indican que las semillas de $S$. stellatus , S. pruinosus y $P$. hollianus contienen alcaloides que podrían resultar tóxicos para las hormigas (Ortega-Nieblas et al., 2001). Desafortunadamente no se cuenta con datos para las semillas del género Escontria y por el momento no es posible conocer si las preferencias de $P$. barbatus podrían relacionarse con el contenido de nutrientes o de compuestos tóxicos de las semillas de esta especie.

Muchos de los estudios sobre remoción de semillas por hormigas han utilizado semillas exóticas y ya se ha demostrado que las tasas de remoción de esas semillas son generalmente más altas que las de semillas nativas (Parmenter $\mathrm{etal}$. 1984; Folgarait \& Sala 2004). En este trabajo utilizamos semillas de cactáceas nativas del Valle de Zapotitlán de las Salinas lo cual nos permite acercarnos al conocimiento de cómo las actividades humanas pueden estar afectando la interacción de la hormiga $P$. barbatus con los recursos disponibles en el sitio. Para un futuro se podrían realizar experimentos con otras semillas además de las de cactáceas, puesto que a pesar de que son especies abundantes en la zona, hay muchas otras plantas como las asteráceas Viguiera dentata y Verbesina, leguminosas como el mezquite Prosopis laevigata y el palo verde Parkinsonia praecox, pastos y muchas otras anuales.

El estudio de la remoción de semillas nativas en las zonas semiáridas intertropicales es un aspecto que aún requiere ser estudiado y el presente trabajo es un primer intento por abordar este tema.

\section{AGRADECIMIENTOS}

Gracias a Silvano Santiago Geraldo "Chimino", guía del Jardín Botánico Helia Bravo Hollis, por acompañarnos a los sitios y por su entusiasmo durante los experimentos de remoción de semillas y al Proyecto PAPCA 2009-2010 otorgado por la Facultad de Estudios Superiores Iztacala, Universidad Nacional Autónoma de México. 


\section{REFERENCIAS}

Brown, J. H., D. W.Davidson, J. C. Munger, \& R. C. Inouye. 1986. Experimental community ecology: the desert granivore system,. In: J. L. Diamond, T. J. Case (eds.), Community ecology. pp 41-61. Harper and Row, New York.

Carroll, C.T \& D.H. Janzen. 1973. Ecology of foraging by ants. Annual Review of Ecology and Systematics 4: 231-257.

Dávila, P., J.L. Villaseñor, R. Medina, A. Ramírez, A. Salinas, J. Sánchez-Ken \& P. Tenorio. 1993. Listados florísticos de México. X. Flora del valle de Tehuacan-Cuicatlán. Instituto de Biología, Universidad Nacional Autónoma de México, México, D.F. pp

Folgarait, P. \& O. Sala. 2004. Granivory rates by rodents, insects, and birds at different microsites in the Patagonian steppe. Ecography 25: 417-427.

Gaytán, S. 2011. Evaluación ecológica de las terrazas aluviales del Valle de Zapotitlán de las Salinas, Puebla. Tesis de Licenciatura. Facultad de Estudios Superiores Iztacala, Universidad Nacional Autónoma de México. Pp 35

Godínez-Alvarez, H. \& A. Valiente-Banuet. 1998. Germination and early seedling growth of Tehuacan Valley cacti species: the role of soils and seed ingestion by dispersers on seedling growth. Journal of Arid Environmnets 39: 21-31

Gordon, D.M. 2002. The regulation of foraging activity in red harvester ant colonies. American Naturalist 159: 509-518

Guzmán, R. 2004. Patrones de actividad de forrajeo de Pogonomyrmex barbatus en el valle semiárido intertropical de Zapotitlán Salinas, Puebla. Tesis de Maestría en Biología. Universidad Autónoma Metropolitana. México

Guzmán-Mendoza, R., G. Castaño \& Herrera-Fuentes, M. C. 2010. Variación espacial y temporal de la diversidad de hormigas en el Jardín Botánico del valle de Zapotitlán de las Salinas, Puebla. Revista Mexicana de Biodiversidad 81: 427-435

Hensen, I. 2002. Seed predation by ants in south-eastern Spain (Desierto de Tabernas, Almería). Anales de Biología 24: 89-96.

Herrick,J., J.E. Van Zee, K.M.Havstat, L. Burket \& W.G.Whitford. 2005. Monitoring manual for grassland, shrubland and savanna ecosystems. Volume II: Design, supplementary methods and interpretation. USDA-ARS Jornada Experimental Range. Las Cruces, New Mexico.

Hölldobler, B. \& E.O. Wilson. 1990. The ants. Belknap Press. Cambridge Massachussets.

Ibáñez J. \& P. Soriano. 2004. Hormigas, aves y roedores como depredadores de semillas en un ecosistema semiárido andino de Venezuela. Ecotropicos 17:38-51

Kaspari, M. 1996. Worker size and seed size selection by harvester ants in a Neotropical forest. Oecologia 105: 397-404

Kelrick, M., J. Macmahon, R. Parmenter \& D. Sisson. 1986. Native seed preferences in shrubsteppe rodents, birds and ants: the relationship of seed attributes and seed use. Oecologia 68:327-337

Kerley G.I.H. \& W.G. Whitford. 2000. Impact of grazing and desertification in the Chihuahuan Desert: plant communities, granivores and granivory. American Midland Naturalist 144 78-91. 
Lomov, B., D.A. Keith \& D.F. Hochuli. 2009. Linking ecological function to species composition in ecological restoration: Seed removal by ants in recreated woodland. Austral Ecology 34:751-760.

López, F., J.M. Serrano \& F.J. Acosta, 1992. Temperature-vegetation structure interaction: the effect on the activity of the ant Messor barbarus (L.). Vegetatio 99-100: 119-128.

López-Galindo, F., D. Muñoz-Iniestra, M. Hernández-Moreno, A. Soler Aburto, M.C. Castillo-López \& I. Hernandez-Arzate. 2003. Análisis integral de la toposecuencia y su influencia en la distribución de la vegetación y la degradación del suelo en la Subcuenca de Zapotitlán Salinas Puebla. Boletín de la Sociedad Geológica Mexicana 56: 19-41.

MacKay, W.P. \& E.E. MacKay, 1989. Diurnal foraging patterns of Pogonomyrmex harvester ants (Hymenoptera: Formicidae). Southwestern Naturalist 34: 213-218.

Marone, L., J.López de Casenave \& V.R. Cueto. 2000. Granivory in Southern South American Deserts: conceptual issues and current evidence. BioScience 50: 123-132.

Muñoz-Iniestra, J.D. 2008. Monitoreo de propiedades físicas y químicas de un suelo aluvial de un ambiente semiárido del sur de México para la búsqueda de indicadores que se relacionen con el estado de conservación y/o degradación del suelo. Tesis de doctorado. Posgrado en Geografía. Universidad Nacional Autónoma de México.

Mull, J.F. \& J.A. MacMahon. 1997. Spatial variation in rates of seed removal by harvester ants (Pogonomyrmex occidentalis) in a shrub steppe ecosystem. American Midland Naturalist 138: 1-13.

Nicolai, N., J.L. Cook \& F.E. Smeins. 2007. Grassland Composition Affects Season Shifts in Seed Preference by Pogonomyrmex barbatus (Hymenoptera: Myrmicinae) in the Edwards Plateau, Texas. Environmental Entomology 36: 433-440.

Oliveros, G.O. 2000. Descrpción estructural de las comunidades vegetales en las terrazas fluviales del Rio Salado en el Valle de Zapotitlán de las Salinas, Puebla, México. Tesis de Licenciatura en Biología. Escuela Nacional de Estudios Profesionales Iztacala, Universidad Nacional Autónoma de México.

Ortega-Nieblas, M., F. Molina-Freaner, M. Robles-Burgueño \& L. Moreno-Vásquez. 2001. Proximate Composition, Protein Quality and Oil Composition in Seeds of Columnar Cacti from the Sonoran Desert. Journal Food Composition Analysis 14: 575-584.

Osorio, B. O., A. Valiente-Banuet, P. Dávila \& R. Medina. 1996. Tipos de vegetación y diversidad B en el Valle de Zapotitlán de las Salinas, Puebla, México. Boletín de la Sociedad Botánica de México 59: 35-58.

Parmenter, R. R., J.A. MacMahon, \& S. B. Vander Wall. 1984. The measurement of granivory by desert rodents, birds and ants: a comparison of an energetics approach and a seeddish technique. Journal of Arid Environments 7: 75-92.

Pirk, G.I.2002. Dieta delas hormigas granívoras Pogonomyrmex prontalisy de Pogonomyrmex rastratus en el Monte central. Tesis de Licenciatura. Universidad de Buenos Aires.

Pulliam, H. R. \& M.R. Brand. 1975. The production and utilization of seeds in plains grassland of southeastern Arizona. Ecology 56: 1158-1166. 
Ríos-Casanova, L. 2005. Efecto de la heterogeneidad ambiental de un abanico aluvial sobre la comunidad de hormigas en San Rafael Coxcatlán, Valle de Tehuacán. Tesis de Doctorado. Doctorado en Ciencias Biológicas, Universidad Nacional Autónoma de México.

Ríos-Casanova, L., A. Valiente-Banuet, A. \& V. Rico-Gray. 2004. Las hormigas del Valle de Tehuacán (Hymenoptera: Formicidae): una comparación con otras zonas áridas de México. Acta Zoológica Mexicana (Nueva serie) 20: 37-54.

Ríos-Casanova,L., A. Valiente-Banuet \& V. Rico-Gray.2006. Ant diversity and its relationship with vegetation and soil factors in an alluvial fan of the Tehuacán Valley, Mexico. Acta Oecologica 29: 316-323.

Sassi, P.L., P.A. Taraborelli, C. E. Borghi \& R. A. Ojeda. 1996. The effect of grazing on granivory patterns in the temperate Monte Desert, Argentina. Acta Oecologica 29: 301-304

Serrano-Cardozo, V.H., J.A. Lemos-Espinal \& G.R. Smith. 2008. Comparative diet of three sympatric Sceloporus in the semiarid Zapotitlan Valley, Mexico. Revista Mexicana de Biodiversidad 79: 1870-3453.

Valiente, B.L. 1991. Patrones de precipitación en el Valle semiárido de Tehuacán, Puebla, México. Tesis de Licenciatura. Facultad de Ciencias, UNAM, México, 61 pp.

Wilby, A. and M.Shachak, 2000. Harvester ant response to spatial and temporal heterogeneity in seed availability: patterns in the process of granivory. Oecologia 125: 495-503.

Whitford, W.G. 2002. Ecology of desert systems. Academic Press. San Diego, Cal. 343 pp.

Whitford, W.G. and G. Ettershank, 1975. Factors affecting foraging activity in Chihuahuan desert harvester ants. Environmental Entomology 4: 689-696.

Zar, J. H. 1996. Biostatistical analysis, 3rd ed. Prentice Hall, Englewood Cliffs, NJ. 
